\title{
Activating the ubiquitin family: UBA6 challenges the field
}

\author{
Marcus Groettrup ${ }^{1}$, Christiane Pelzer ${ }^{1}$, Gunter Schmidtke ${ }^{1}$ and Kay Hofmann ${ }^{2}$ \\ ${ }^{1}$ Division of Immunology, Department of Biology, University of Constance, D-78457 Konstanz, Germany \\ ${ }^{2}$ Miltenyi Biotec GmbH, Bioinformatics, D-50829 Köln, Germany
}

\begin{abstract}
Since its discovery in 1981, ubiquitin-activating enzyme 1 was thought to be the only E1-type enzyme responsible for ubiquitin activation. Recently, a relatively uncharacterized E1 enzyme, designated ubiquitin-like modifier activating enzyme 6 , was also shown to activate ubiquitin. Ubiquitin-activating enzyme 1 and ubiquitin-like modifier activating enzyme 6 are both essential proteins, and each uses a different spectrum of ubiquitin-conjugating (E2) enzymes. Ubiquitin-like modifier activating enzyme 6 activates not only ubiquitin, but also the ubiquitin-like modifier FAT10 (human leukocyte antigen F-associated transcript 10), which, similarly to ubiquitin, serves as a signal for proteasomal degradation. This new layer of regulation in ubiquitin activation markedly increases the versatility of the ubiquitin conjugation system.
\end{abstract}

\section{The ubiquitin family}

The post-translational modification of proteins by phosphate, sugars or lipids has long been recognized as a means to alter the properties of proteins. When the ATPdependent degradation of proteins was first investigated nearly three decades ago, it became clear that the scope of protein modifications extends to the covalent linkage with a 76-residue polypeptide termed ubiquitin [1,2]. The ubiquitin $\mathrm{C}$ terminus becomes isopeptide-linked to the $\varepsilon$ amino group of lysines within target proteins. Ubiquitin itself is then ubiquitylated on K48, leading to the assembly of ubiquitin chains. Chains of four K48-linked ubiquitins or longer are bound by $26 \mathrm{~S}$ proteasome subunits, leading to target protein degradation following the removal and recycling of ubiquitin [3]. Ubiquitin chains can not only be linked through lysine 48 , but also through the other seven lysines within ubiquitin. Chains linked through K63, for example, have a role in endocytosis and signal transduction through nuclear factor- $\mathrm{B}[4,5]$. The conjugation and deconjugation of ubiquitin falls under stringent enzymatic control. Initially, ubiquitin requires activation by a ubiquitin-activating enzyme (E1) that uses ATP and its active site cysteine to form a thioester with the ubiquitin $\mathrm{C}$ terminus [6]. Then ubiquitin is transferred to the active site cysteine of a ubiquitin-conjugating enzyme (E2). A ubiquitin-charged E2 enzyme and a substrate are finally bound by a ubiquitin ligase (E3) that mediates the isopeptide linkage of the activated ubiquitin to the substrate (Figure 1).

Corresponding author: Groettrup, M. (Marcus.Groettrup@uni-konstanz.de).
Ubiquitin was originally described as the heat-stable polypeptide of the ATP dependent proteolytic system' [1]. Ubiquitin is stable because it folds tightly in a so-called ' $\beta$ grasp fold', wherein four $\beta$-sheets pack around a central $\alpha$-helix. Ubiquitin-protein binding introduces a new, separately folded domain to the protein which enables binding of new interaction partners that bear one or more of the numerous types of ubiquitin-interaction domains [7]. It is now apparent that binding to proteasomal subunits or docking proteins is only one example of ubiquitin-mediated interactions.

Twenty years after the first reports on ubiquitin, it became clear that ubiquitin is only the tip of an iceberg because nearly a dozen ubiquitin-like proteins use the same biochemical principles [8]. Although not all ubiquitin-like proteins share significant sequence identity with ubiquitin, each possesses the typical ' $\beta$-grasp' or 'ubiquitinfold' structure. The ubiquitin-like proteins can be subdivided into the 'ubiquitin-domain proteins', which contain ubiquitin-like domains as protein-protein interaction domains but do not become conjugated to target proteins, and the so-called 'ubiquitin-like modifiers' (ULMs), which become isopeptide linked to selected target proteins through dedicated E1-E2-E3 enzyme cascades. To date, the ULMs small ubiquitin-related modifier (SUMO)1-3, neuronal precursor cell-expressed developmentally downregulated protein (NEDD)8, interferon-stimulated gene (ISG)15, autophagy-related (ATG)8, ATG12, human leukocyte antigen F-associated transcript (FAT)10, ubiquitinrelated modifier (URM)1, ubiquitin-fold modifier (UFM)1 and monoclonal nonspecific suppressor factor (MNSF) $\beta$ have been described [8]. Each ULM contains one or two glycines at the $\mathrm{C}$ terminus of its ubiquitin-fold domain that is used to form the isopeptide bond with target proteins, and most (except for FAT10, ATG12 and URM1) are synthesized as precursors whose conjugation requires C-terminal cleavage at this glycine through specific processing proteases. Again, with the exception of FAT10 [9], none of the ULMs primarily function in proteolytic targeting, although recent work demonstrated that polyubiquitylation and/or accelerated degradation can be a secondary consequence of SUMO-1, and possibly NEDD8, modification [10-13]. Instead, modification of proteins with ULMs directs changes in other properties, including intracellular localization, enzymatic turnover, signal transduction competence and transcriptional activity [8]. Remarkably, each ULM requires specific E1, E2 and E3 enzymes, indicating that separate control of these path- 


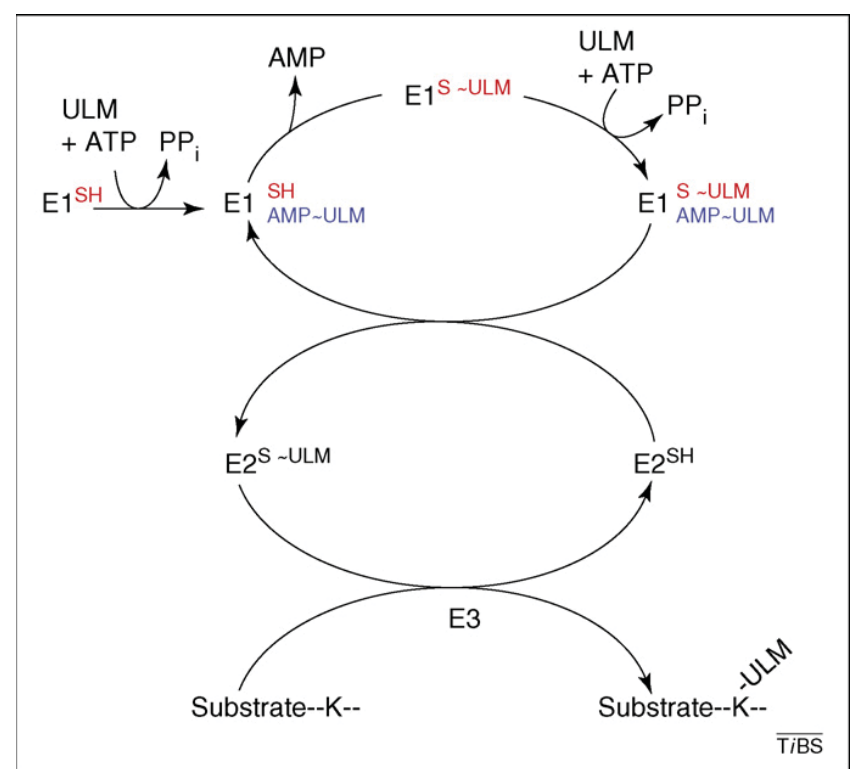

Figure 1. Activation of an ULM by an E1 enzyme. The E1 enzyme can bind to the modifier at two different sites, an adenylation site (blue) and a site where the modifier is thioester linked to the E1 (red). First, the E1 enzyme uses ATP to adenylate, and thereby activate, the ULM. The activated ULM is then transferred from the E1 ThiF domain to the active site cysteine-containing domain to form a thioester Subsequently, a second ULM binds and is adenylated. The formation of the thioester enhances the affinity for E2 binding, and the activated ULM is transthiolated to a cognate E2 enzyme. An E3 ligase then binds to the ULM-charged E2 in addition to a substrate, and catalyzes the isopeptide bond formation between the $C$ terminus of the ULM and an $\varepsilon$-amino group of lysine in a substrate protein.

ways has evolved at the step of ULM activation. Until now, the only examples of shared E1 enzymes have been the activation of SUMO1-3 by the heterodimeric E1 enzyme, AOS1-UBA2 (activation of Smt3p 1-ubiquitin-like modifier activating enzyme 2), and the activation of ATG8 and ATG12 by their common E1 enzyme, ATG7 [14]. The recent discovery that ubiquitin can be stimulated by two different E1 enzymes, ubiquitin-activating enzyme 1 (UBE1) and UBA6 [15-17], and that UBA6 can activate two different ULMs (ubiquitin and FAT10) [17], emphasizes that a unilateral assignment of an E1 enzyme to a select ULM is no longer valid. The enormously diverse ubiquitin system does not rely on a single E1 enzyme but, rather, on two. These unexpected findings open new avenues for regulation at the level of activating a ULM, but also pose several questions regarding the specificity of ULM transfer.

\section{Structure and enzymatic mechanisms of E1 enzymes}

To date, eight E1 enzymes have been identified owing to their conserved three-domain structure, and most have been assigned to one of the known ULMs (Table 1). E1 enzymes are either formed by a single polypeptide chain or are heterodimers such as the E1 enzymes AOS1-UBA2 and $\beta$-amyloid precursor protein-binding protein (APPBP)-1-UBA3 for SUMO1-3 and NEDD8, respectively, which carry the characteristic E1 domains on two separate polypeptides $[18,19]$. One task of E1 enzymes is to bind noncovalently to their cognate ULM, thereby ensuring that the appropriate ULM is selected for the respective conjugation cascade. In addition, the ULM $\mathrm{C}$ terminus must be activated so that it can eventually form an isopeptide bond. This activation is achieved through an ordered mechanism in which first ATP is bound by the E1, followed by binding of the ULM $[6,20,21]$ (Figure 1). The ULM glycine carboxy group then attacks the $\alpha$-phosphate of ATP to form an energy-rich acyl-phosphate anhydride with AMP. Subsequently, the activated ULM glycine carboxy group is subject to a nucleophilic attack by the sulfur atom of a cysteine conserved in the active sites of all E1 enzymes. This activity results in the formation of an energy-rich E1ULM thioester, which remains covalently bound while a second ULM is recruited to the adenylation site. A cognate E2 is then bound noncovalently, and the ULM thioester is passed onto the $\mathrm{E} 2$ active site cysteine in a transthiolation reaction. This step ensures that an activated ULM is not transferred onto an inappropriate E2. Finally, the E2ULM thioester is released and the cycle begins again (Figure 1). Interestingly, the recent structural determination of an E1-E2-NEDD8 complex indicated that the formation of the E1-NEDD8 thioester enhances the affinity for the cognate E2 Ubc12, which is lowered again after the transthiolation reaction has occurred, thus facilitating E2-NEDD8 release [22].

The crystal structures have been solved for the NEDD8 and SUMO E1 enzymes in complex with their cognate ULM. A partial structure also exists for UBE1, the ubiquitin E1 enzyme [23-25]. Considering the high degree of conservation among the known E1 enzymes, it can be assumed that all E1 enzymes possess three modular domains, which regulate the functions outlined here. The domains are arranged around two clefts that coordinate nucleotide and protein binding [26]. The N-terminal portion of E1 enzymes directs ATP- and ULM binding. This module is composed of two ThiF-homology motifs, which were named according to a homologous domain in the bacterial enzyme ThiF (Box 1). The second ThiF-homology domain contains the consensus motif GxGxxGCE, which directs ATP binding. The next module bears the active site

Table 1. The eight known human E1 enzymes

\begin{tabular}{|c|c|c|c|c|c|}
\hline Name of E1 & Molecular weight (kDa) & Identity (\%) ${ }^{a}$ & ULM & E2 enzyme & Refs \\
\hline UBE1 & 118 & 100 & Ubiquitin & many & [29] \\
\hline UBE1L & 112 & 46 & ISG15 & UbcH8 & [67] \\
\hline AOS1 (SAE1) & 38 & 30 & SUMO-1,2,3 & UBC9 & {$[68,69]$} \\
\hline UBA2 (SAE2) & 71 & 17 & & & \\
\hline APP-BP-1 & 60 & 17 & NEDD-8 & UBC12 & [70] \\
\hline UBA3 & 51 & 21 & & & \\
\hline UBA4 (MOCS3) & 50 & 17 & URM-1 & Unknown & [71] \\
\hline UBA5 (UBE1DC1) & 45 & 13 & UFM-1 & Ufc1 & [72] \\
\hline ATG7 & 78 & 6 & ATG12, ATG8 & ATG10, ATG3 & [73] \\
\hline
\end{tabular}

aThe amino acid identity to UBE1 is provided. 
cysteine found within the consensus motif PxCTxxxP. The most C-terminal 100 amino acids of E1 enzymes constitute the third module, a ubiquitin-fold domain, which binds to E2 enzymes. Domain-swapping experiments between different E1 enzymes have revealed that this domain accounts for the selectivity of E1 enzymes for their cognate E2s $[16,27]$.

\section{UBA6: a second ubiquitin-activating enzyme}

An E1 enzyme that activates ubiquitin was initially described, characterized and purified in 1981 and 1982 $[20,28,29]$; corresponding genes, named $U B A 1$ in yeast and plants and $U B E 1$ in mammals, were cloned a decade later [30-33]. In Saccharomyces cerevisiae, UBA1 is essential, indicating that no other E1-type enzyme can compensate for this deficiency. In mammals, UBE 1 is encoded on the $\mathrm{X}$ chromosome, which explains how mutations in the single copy of $U B E 1$ generated several temperature-sensitive $(t s)$ cell lines which arrested in the G2-S phase of the cell cycle at a restrictive temperature [31,34-36]. Indeed, transfection of these cells with constructs encoding UBE1 rescued the ubiquitylation defect, indicating that the $t s$ mutation affected $U B E 1$ [37]. These $t s$ cell lines have been used extensively to characterize the function of UBE1 and of the entire ubiquitylation system. In the $t s 85$ cell line, polyubiquitylation and degradation of pulse-labeled proteins is reduced to $<15 \%$ of the level observed in wild-type cells. The low residual ubiquitin activation

\section{Box 1. The origin and evolution of E1 enzymes}

The structural analysis of two proteins from Escherichia coli has revealed their striking similarity to the E1 enzymes that activate ULMs. These two highly conserved proteins are MoeB [52], which is involved in the biosynthesis of molybdopterin, a tricyclic pyranopterin that coordinates molybdenum to build the molybdenum cofactor (Moco), and ThiF [53,54], an enzyme required for thiamine biosynthesis (Figure I). Both enzymes bind to ATP and the ubiquitin-fold proteins MoaD and ThiS, respectively, to form an adenylate similar to the ULM adenylates formed by E1 enzymes. The ATP-binding domain is conserved between MoeB and ThiF in structure and sequence $144 \%$ identity) but also between these two proteins and the corresponding adenylation domains of E1 enzymes ( 20-30\% identity; Figure II). Therefore, these domains have been termed ThiF homology domains. Although MoeB and ThiF function as homodimers in which each monomer contains one ATP-binding ThiF homology domain, the $\mathrm{N}$ termini of E1 enzymes contain two ThiF homology units, which together bind only to one ATP molecule. Similar to E1 enzymes, MoeB and ThiF use the adenylates of their cognate ubiquitin-fold proteins
MoaD and ThiS to form thioesters with a cysteine of the activating enzymes, with the only exception being that the thiocarboxylate of ThiS is disulfide linked to ThiF (Figure I). In contrast to the transfer of thioester-linked ULMs between E1 and E2 enzymes, MoaD and ThiS are released after activation as thiocarboxylates, which serve as sulfur donors for the biosynthesis of molybdopterin and thiamine, respectively. This process requires interaction with further factors $[53,54]$, which might explain why the active site cysteine-containing domains of E1 enzymes, on the one hand, and MoeB and ThiF, on the other hand, are less conserved.

Although the structures of the ubiquitin-fold proteins MoaD and ThiS are well conserved compared with ULMs [55,56], their sequences are not, except for a common diglycine motif at the $C$ terminus of MoaD and ThiS. The only ULM that has significant sequence identity to MoaD or ThiS is Urm1 (Figure II), consistent with the notion that ULMs, MoaD and ThiS have evolved from common ancient sulfur carrier proteins, and that Urm1 represents an evolutionary link between the two groups.

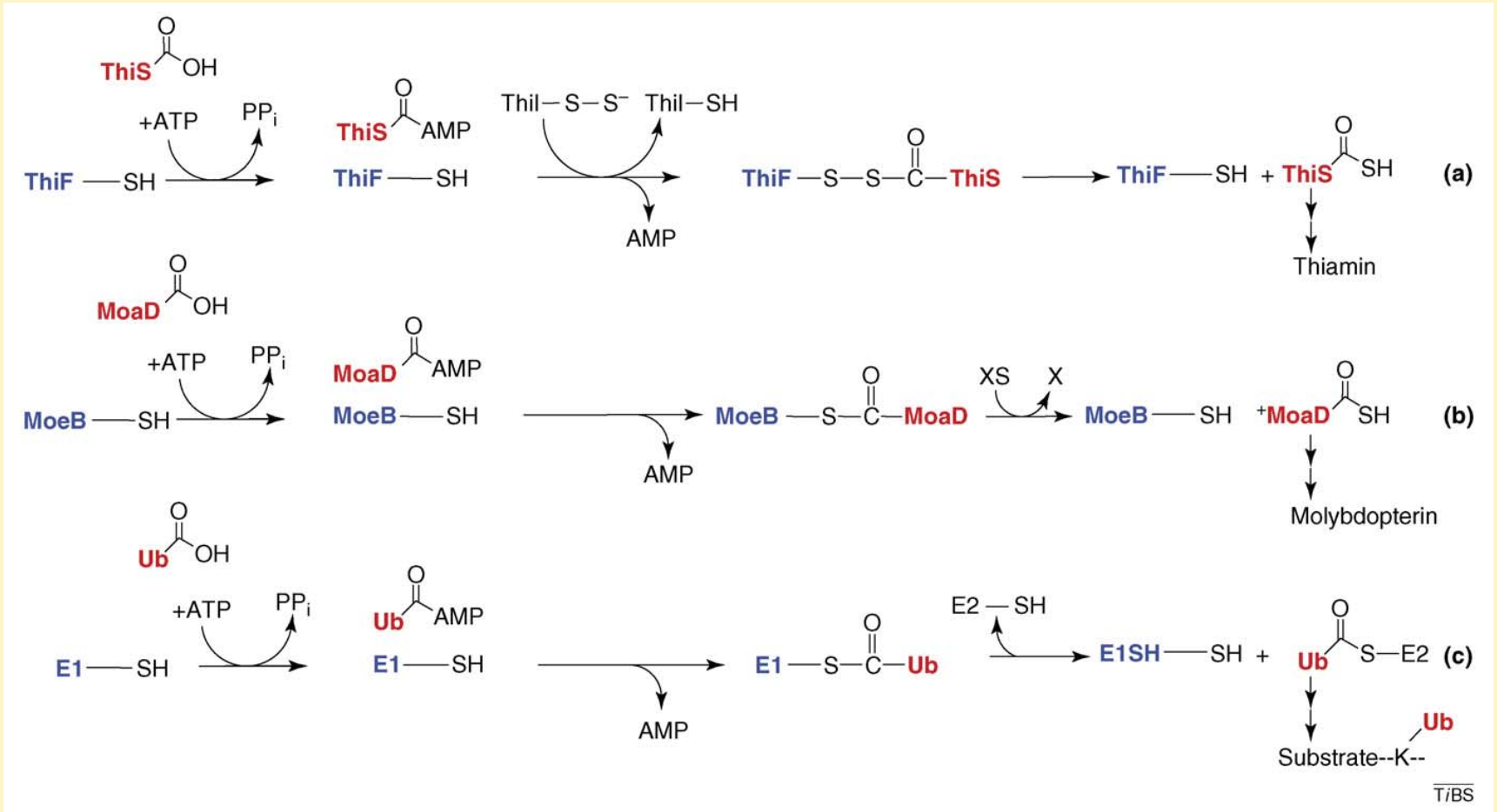

Figure I. Parallels in the activation of the ubiquitin-fold proteins ThiS, MoaD and ubiquitin. The top row (a) shows the formation of a ThiS adenylate by the activating enzyme ThiF, which is involved in thiamine biosynthesis in $E$. coli. The thiocarboxylate of ThiS serves as a sulfur donor for the synthesis of thiamin. The middle row (b) shows MoaD adenylation by the activation enzyme MoeB, which together constitute the molybdopterin synthase. The MoaD thiocarboxylate contributes the sulfur atoms that bind to molybdenum in the molybdenum cofactor. The bottom row (c) shows the activation of ubiquitin (Ub) and transfer onto a lysine residue (K) within a substrate protein. The activation enzymes that contain the ThiF homology motives are displayed in blue, and the ubiquitin-fold proteins in red. 


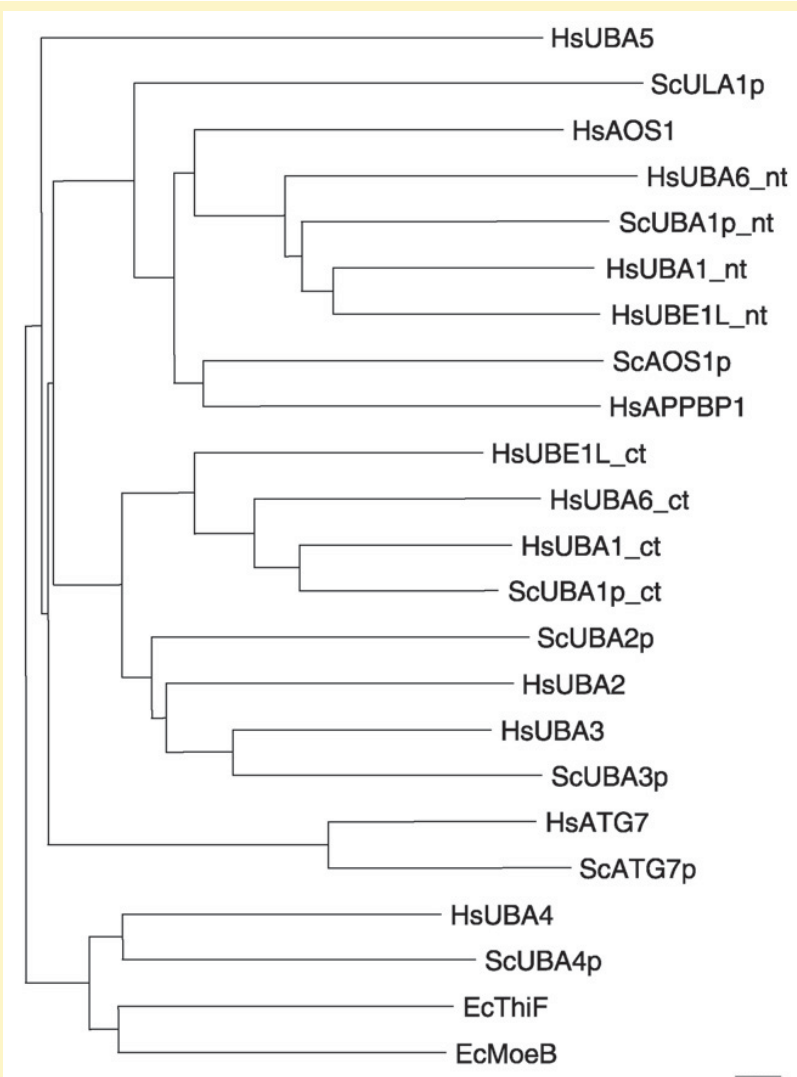

Figure II. Dendrogram analysis of the evolution of human (Hs) and yeast (Sc) E1 enzymes. The dendrogram was calculated by the 'neighbor joining' algorithm from a multiple sequence alignment of nucleotide-binding Rossman-fold domains - where possible, with support from 3D structures. Because we consider the prokaryotic MoeB and ThiF enyzmes and their eukaryotic counterpart UBA4 as the most ancient E1s, they can be used as an outgroup, resulting in a pseudo-rooted tree. The Nterminal (nt) and C-terminal (ct) Rossman-fold domains of UBE1, UBA6 and UBE1L were separately aligned. Sequence conservation is usually higher within the nucleotide-binding C-terminal domain copies. When focusing on this subdomain, UBA6 is the closest relative of UBA1.

was assumed to result from incomplete UBE1 inactivation and was taken as evidence that, in mammals, no other E1 for ubiquitin exists. However, in mice [38] and marsupials [39], a second UBE1-like gene is encoded on the Y chromosome (UBE1y); this is $90 \%$ identical to $U B E 1$ found on the $\mathrm{X}$ chromosome (UBE1x) [40]. Plants also have two or three different $U B E 1$ genes that most likely resulted from gene duplications and are $\sim 90 \%$ identical to each other; their enzymatic activity and the efficiency of ubiquitin transfer to several E2 enzymes is similar [41]. It therefore seemed that UBE1 and these structurally and enzymatically related extra copies of UBE1 were the only ubiquitinactivating enzymes.

It came as a surprise when this notion was proven incorrect. Recently, three groups independently discovered that a relatively uncharacterized gene previously named ubiquitin-activating enzyme E1-like protein 2 (UBE1L2) or monocyte protein-4 (MOP-4), the protein product of which contains all the aforementioned structural elements of E1 enzymes, also activates ubiquitin (Figure 2). This novel E1 enzyme is $42 \%$ identical to UBE1 and $36 \%$ identical to the ISG15 E1 enzyme UBE1L. Pelzer et al. used the name UBE1L2 [15], whereas Jin et al. renamed it UBA6 [16] and Chiu et al. termed the protein E1-L2 [17]. Following the UBA nomenclature (Table 1), we agree with Jin et al. [16] that UBA6 is the most systematic name, and we use UBA6 here.
In a search for the FAT10 E1 enzyme, Pelzer et al. [15] cloned and expressed all eight known or putative human E1 type enzymes and tested whether they were able to form thioesters with recombinant glutathione S-transferase (GST)-ULM fusion proteins. Because no ULM was known to be activated by UBA6, these authors tested all GST-ULM fusion proteins and, surprisingly, found that UBA6 activated GST-ubiquitin as efficiently as UBE1 in vitro and also in intact cells [15]. In the GST pull-down assay, no other GST-ULM fusion proteins formed covalent conjugates that were sensitive to reducing agents, initially indicating that UBA6 was specific for ubiquitin. Next, these authors, in cooperation with Scheffner's group, investigated whether ubiquitin activated by UBA6 could be transferred to E2 and E3 enzymes and become isopeptide linked to a substrate [15]. Indeed, UBA6 was as efficient as UBE1 in transferring activated ubiquitin onto the broadspectrum E2 enzyme UbcH5b and further onto the E3 enzymes HectH9 and E6-AP. Moreover, the activated ubiquitin could become isopeptide linked to the ubiquitylation substrate p53 under the catalysis of the E3 ligase mouse double minute (Mdm)2 [15] (Figure 2).

The activation of one member of the ubiquitin family by two different $\mathrm{E} 1$ enzymes is a new paradigm and poses the interesting question of why two enzymes have evolved for apparently the same purpose. A mere redundancy does not seem to apply because $U B A 6$ deletion in the mouse leads to 


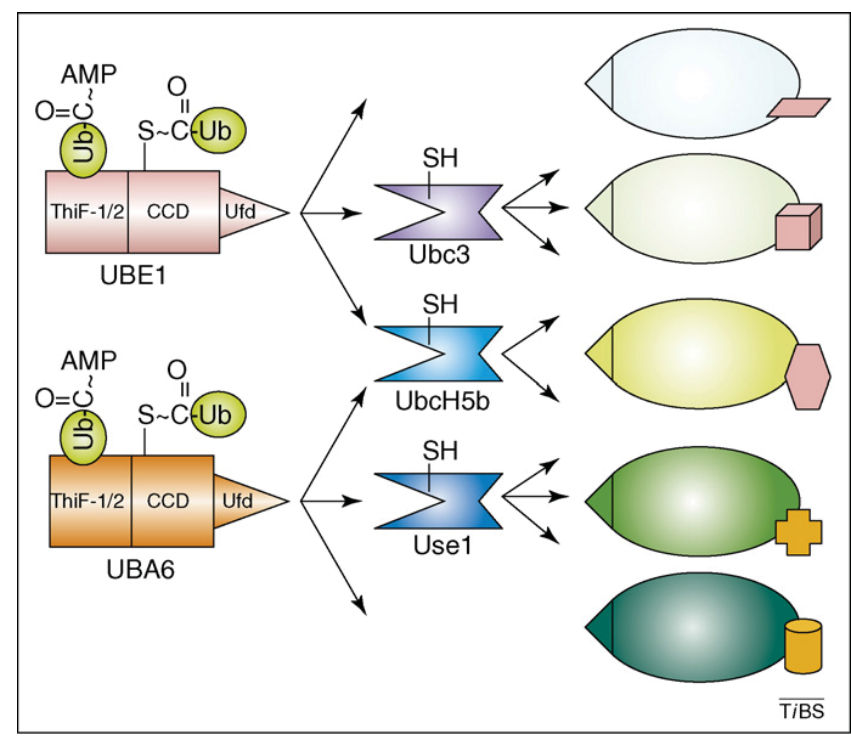

Figure 2. Cooperation of UBE1 and UBA6 with different E2 enzymes. UBE1 (red) and UBA6 (orange) load ubiquitin (Ub; green) onto a different spectrum of E2 enzymes and might therefore initiate the ubiquitylation of different substrates. Both UBE1 and UBA6 consist of (i) two N-terminal ThiF homology units which adenylate ubiquitin, (ii) a central active site cysteine-containing domain (CCD) which forms a thioester with ubiquitin, and (iii) a C-terminal ubiquitin-fold domain (Ufd) which binds to E2 enzymes. As examples, the E2 enzyme Use1 (blue) accepts ubiquitin only from UBA6; the E2 enzyme Ubc3 (purple) accepts ubiquitin only from UBE1; and the E2 enzyme UbcH5b (turquoise) can be charged with ubiquitin by both UBE1 and UBA6. E3 enzymes (depicted in a spectrum of green colors) selectively bind to E2 enzymes and their cognate substrates (small symbols attached to the bottom right of the E3) to transfer ubiquitin activated by UBE1 (red substrates) or UBA6 (orange substrates).

embryonic lethality before day E10.5 [17]. One possibility is that UBA6 has different enzymatic properties than UBE1, although this is not likely because the kinetics of ubiquitin activation and the affinity for ubiquitin do not differ between UBE1 and UBA6 [16]. An intriguing possibility is that UBA6 and UBE1 might use different E2 enzymes, which in turn cooperate with different E3 enzymes to ubiquitylate a unique subset of protein substrates. This scenario would endow the ubiquitylation system with a new layer of regulation at the level of ubiquitin activation. Jin et al. [16] undertook the impressive effort of testing 29 different E2 enzymes for their ability to accept activated ubiquitin from either UBE1 or UBA6. They found that the $29 \mathrm{E} 2$ enzymes could be grouped into four classes: 14 E2s were charged by UBE1 but not by UBA6, nine E2s were charged equally by UBE1 and UBA6, five E2 enzymes could be charged by neither UBE1 nor UBA6 and, most interestingly, one relatively uncharacterized E2 enzyme, UBE2Z [42], was only activated by UBA6 but not by UBE1, which led to the suggestion that this enzyme be renamed 'UBA6-specific E2' (Use1). The selectivity for certain E2 enzymes was also demonstrated by Chiu et al. [17]; they showed that Ubc3 and E2-25K could accept ubiquitin from UBE1 but not from UBA6. These assignments of cooperating E1 and E2 enzymes were all based on qualitative assays with a single endpoint, and need to be confirmed using quantitative enzymological data. However, some of the in vitro findings were further corroborated by knockdown experiments because Cdc34A and Cdc34B (i.e. two enzymes that were selectively charged by UBE1 in vitro) also relied on UBE1 but not on UBA6, in intact cells. Conversely, knockdown of UBA6, but not of UBE1, interfered with the in vivo loading of Use1 with activated ubiquitin [16]. It is therefore logical to conclude that UBE1 and UBA6 only partially overlap with respect to their spectrum of $\mathrm{E} 2$ enzymes, and it is reasonable to expect that this specificity will extend to E3 enzymes and their cognate substrates (Figure 2).

Other characteristics that might differ between UBE1 and UBA6 are their expression profiles in phylogeny, ontogeny or in different organs. UBA6 is expressed in vertebrates and the sea urchin but is not detected in insects, worms, fungi or plants. UBA6 expression during embryonal development has not yet been reported but the early lethality of the conventional $U B A 6$ knockout mice indicates that it is expressed in mice before day E10.5. When UBA6 mRNA expression was quantitatively assessed in 11 different mouse organs, a fivefold higher expression was found in the testis compared with all other organs, which expressed low levels of UBA6 [15]. This finding might point at an enhanced need for UBA6 activity during spermatogenesis and would be consistent with the UBA6-dependent ubiquitylation of testis-specific target proteins. A testis-specific E2 enzyme [43], E3 enzyme [44] and ubiquitin-specific protease [45] have been described. The existence of a second UBE1 enzyme encoded on the $\mathrm{Y}$ chromosome of mice and marsupials argues for an enhanced need for ubiquitin activation during spermatogenesis because the differentiation from spermatocytes to mature elongated spermatids involves condensation and removal of much of the cytoplasm and the endoplasmic reticulum. Nevertheless, other than the strikingly enhanced expression in the testis, UBA6 is broadly expressed in all tissues at a low level. Jin et al. [16] compared UBE1 and UBA6 expression levels, by western blot analysis, in a panel of cell lines, and estimated the UBA6 expression levels to be at least tenfold lower than those of UBE1. Interestingly, the frequently used $t s U B E 1$ mutant cell lines $t s 85$ and $t s 20$ expressed normal UBA6 levels, which opens the possibility that the residual level of high molecular mass polyubiquitin conjugates found in these cells might result from unaltered UBA6 expression rather than the previously proposed incomplete heat inactivation of mutant UBE1. This finding has the important implication that the degradation of specific protein substrates that occurs at the restrictive temperature in these cell lines might not be independent of ubiquitin activation in general but could instead rely on ubiquitin activation by UBA6 rather than by UBE1.

\section{UBA6 activates two different modifiers: ubiquitin and FAT10}

UBA6 challenges a second major paradigm in the ubiquitin field - namely, that a specific E1 enzyme only activates one type or one closely related subfamily of modifiers. Thoroughly investigated examples for this selectivity are NEDD8 and ubiquitin, which are nearly $60 \%$ identical; a single arginine residue in the E1 for NEDD8 determines its selectivity for NEDD8 and prevents aberrant ubiquitin activation [46]. By contrast, UBA6 was recently shown to activate two ubiquitin family proteins, ubiquitin and FAT10 [17] (Box 2). Therefore, UBA6 is another example of an E1 that activates two structurally and functionally 


\section{Box 2. FAT10: the youngest member of the ULM family}

When the MHC class I locus was sequenced in 1996, FAT10 was identified as encoding an $18 \mathrm{kDa}$ protein which comprises two ubiquitin-like domains in tandem array [57] (Figure I). In evolutionary terms, FAT10 is the youngest member of the ULM family, being expressed only in mammals. FAT10 is constitutively expressed in mature dendritic cells and B cells but is synergistically induced in many tissues by the proinflammatory cytokines IFN- $\gamma$ and TNF- $\alpha$ $[47,49,58]$. FAT10-deficient mice are viable and fertile but they are hypersensitive to lipopolysaccharide [59]. These properties point to a possible function for FAT10 in the immune system. Ectopic expression of FAT10 induces caspase-dependent apoptosis in murine fibroblasts [50] and renal tubular epithelial cells [60]. In seeming contradiction to this function, FAT10 is often overexpressed in several carcinomas, most notably in hepatocellular carcinoma [48]. This finding, along with the observed chromosome missegregation in tumor cell lines overexpressing FAT10 [61], was attributed to the noncovalent interaction of FAT10 with the spindle assembly checkpoint protein mitotic arrest deficiency 2 (MAD2) [47]. Moreover, p53 has been reported to direct the downregulation of FAT10 transcription [62]. Whether FAT10 expression contributes to tumor development or is a consequence of IFN stimulation in the tumor requires further investigation.

FAT10, similarly to ubiquitin, serves as a signal for proteasomal degradation. The fusion of long-lived proteins (e.g. green fluorescent protein or dihydrofolate reductase) to FAT10 causes their rapid degradation by the proteasome; this function is independent of the ubiquitin system [9]. Because no evidence for the deconjugation of FAT10 from its substrates has been obtained, FAT10 is probably degraded, along with its substrates, in a manner similar to that seen with ubiquitin-modified substrates when deconjugation is inhibited [3]. The FAT10 degradation rate is markedly enhanced by the coexpression of NEDD8 ultimate buster 1 long (NUB1L) [63], an IFNinducible protein that noncovalently associates with FAT10 through three C-terminal UBA domains and with the $26 \mathrm{~S}$ proteasome through an N-terminal ubiquitin-like domain. The NUB1L ubiquitin-like domain, but not FAT10 binding, is essential for enhancing FAT10 degradation [64]. Because FAT10 itself binds to the proteasome, we propose that NUB1L binds to a different site at the $26 \mathrm{~S}$ proteasome, thus inducing a conformational change that results in rapid degradation of FAT10 and FAT10-conjugated proteins. (a) Ubiquitin

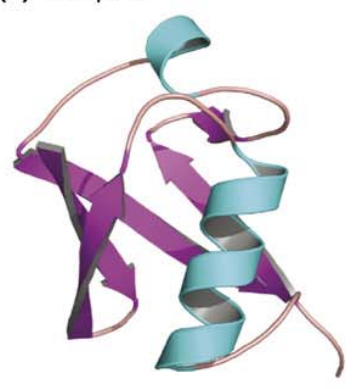

(b) FAT10 (model)

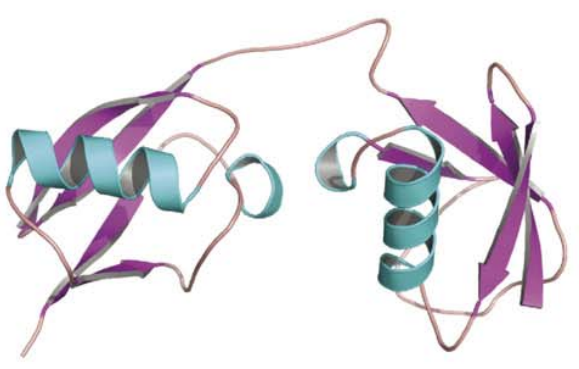

$\overline{\mathrm{T} i \mathrm{BS}}$

Figure I. Ribbon diagrams of the ubiquitin structure and the FAT10 model structure. Ubiquitin and the N-and C-terminal ubiquitin-like domains of FAT10 show the typical $\beta$-grasp fold, where a central $\alpha$-helix (turquoise) is surrounded by $\beta$-sheets (purple). The diagrams were generated with the Pymol program, using the Research Collaboratory for Structural Bioinformatics (RCSB) Protein Data Bank entry 1UBO for human ubiquitin [65] and 1Z2M for ISG15 [66] that was predicted by the Phyre server as a model for the human FAT10 primary structure. This model was previously proposed because the residues making contacts between the $3_{10}$ helices of the two $\beta$-grasp folds in ISG15 and FAT10 are conserved [66]. However, because the linker between the two ubiquitin folds of FAT10 is hydrophilic and probably flexible, a different orientation between these two domains in solution is possible.

unrelated modifiers (Figure 3). Chiu et al. [17] demonstrated that FAT10 is activated by UBA6, but not by $\mathrm{UBE} 1$ or UBE1L, in vitro; this activation relied on the UBA6 active site cysteine and the FAT10 C-terminal diglycine motif. Initially, the activation of GST-FAT10 by UBA6 in GST pull-down experiments could not be detected [15] but the use of different tags enabled the activation to be consistently observed [17]. In HEK293 cells, endogenously expressed UBA6 and FAT10 are known to be covalently bound because FAT10 is released from UBA6 immunoprecipitates following dithiothreitol treatment. Chiu et al. also addressed whether UBA6 knockdown in HEK293 cells would abolish FAT10 conjugate formation. Previously, it had been difficult to detect FAT10 conjugates in HEK293, COS or HeLa cell lines $[9,47,48]$ (Box 2). However, following overexpression from a His $_{6}-\mathrm{FLAG}_{3}-\mathrm{FAT} 10$ expression construct, Chiu et al. [17] found an array of faint putative FAT10 conjugates in HEK293 cells that did not appear when a C-terminal diglycine mutant of FAT10 was expressed. These conjugates were strongly, but not completely, diminished following UBA6 knockdown but not knockdown of UBE1. This finding was interpreted to indicate that UBA6 is both necessary and sufficient to activate FAT10, a conclusion which should be confirmed in cells treated with the FAT10inducing proinflammatory cytokines interferon (IFN)- $\gamma$ and tumor necrosis factor (TNF)- $\alpha$ [49].

If UBA6 activates both ubiquitin and FAT10, how do these modifiers compete for activation? Chiu et al. [17] showed that even a fourfold molar excess of FAT10 over ubiquitin in vitro still enabled only marginal formation of UBA6-FAT10 complexes; however, these were readily apparent in the absence of competing ubiquitin. Assuming that the ubiquitin and FAT10 proteins used in this assay were intact, this result indicates that UBA6 preferentially activates ubiquitin. The solution to this problem could lie in the strong and rapid FAT10 induction following IFN- $\gamma$ and TNF- $\alpha$ stimulation [49] (Figure 3), which might shift the equilibrium in favor of FAT10 conjugation. Alternatively, post-translational modifications of FAT10, which have been detected on two-dimensional gels [50], might positively affect FAT10 activation in cells. Another interesting question is whether the group of E2 enzymes that can be charged by UBA6-ubiquitin will also accept FAT10. Chiu et al. [17] started to address this question and found that two E2 enzymes, UbcH5 and Ubc13, which can be 


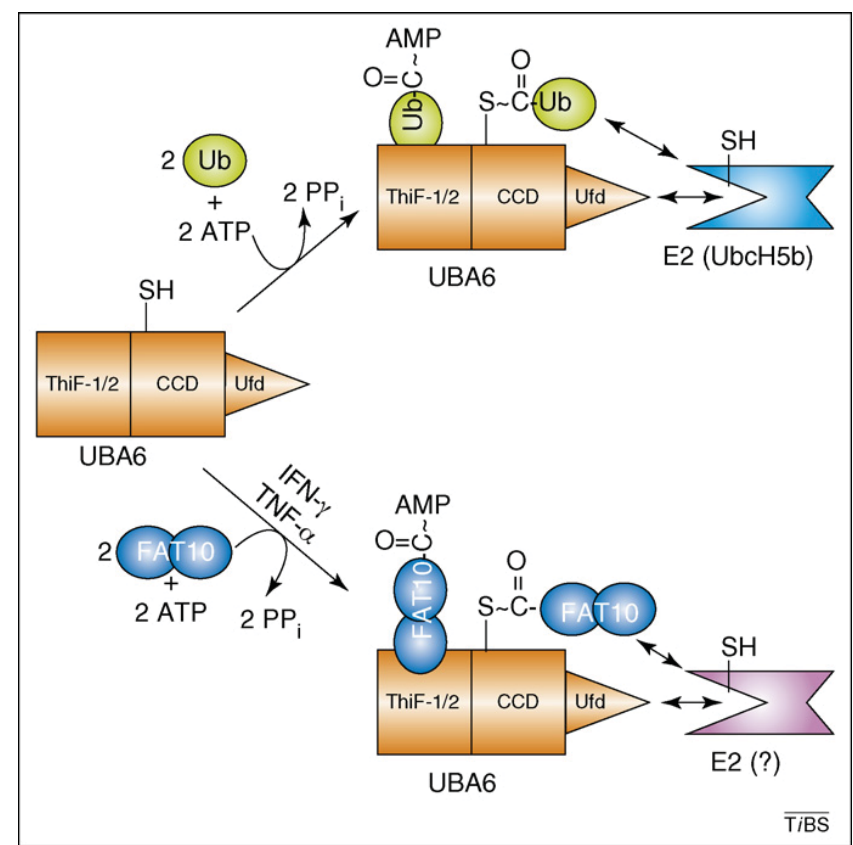

Figure 3. UBA6 activates both ubiquitin and FAT10. In vitro, the UBA6-mediated activation of ubiquitin (Ub; green) is preferred over FAT10 (blue). However, when FAT10 is strongly induced by the proinflammatory cytokines IFN- $\gamma$ and TNF- $\alpha$, FAT10 can compete with ubiquitin for activation by UBA6. We postulate that the UBA6 ubiquitin-fold domain (Ufd; orange) and the ULM codetermine the E2-type enzyme to which the respective ULM is transferred [e.g. ubiquitin to $\mathrm{UbcH} 5 \mathrm{~b}$ (turquoise) and FAT10 to a still unknown E2 (pink)].

ubiquitin charged by UBA6, did not accept FAT10 in vitro. This finding indicates that in addition to the E1 ubiquitin fold domain, the ULM itself codetermines its transferability to a given E2 (Figure 3). Similarly, it is interesting that Ubc9, the cognate E2 for SUMO, through noncovalent SUMO-1 binding, facilitates the assembly of SUMO-1 chains [51]. It might therefore be the case that noncovalent FAT10 binding to the still elusive E2 enzyme(s) of FAT10 contributes to the specificity of the FAT10 conjugation cascade.

\section{Concluding remarks and future perspectives}

The unique properties of UBA6 challenge two major paradigms of ULM conjugation: first, UBE1 and UBA6 are the first examples of E1 enzymes that both activate the same member of the ULM family, and, second, UBA6 is, aside from ATG7 and AOS1-UBA2, the third example of an E1 that activates two ULMs: ubiquitin and FAT10, which have widely different structural and biological properties. Because UBE1 and UBA6 cooperate with only partially overlapping pools of E2 enzymes [16,17], it is expected that at the stage of ubiquitin activation, a new level of control exists for distinguishable E1-E2-E3-substrate cascades. To substantiate this hypothesis, it will be necessary to identify further E2 enzymes, E3 enzymes and substrates that rely on UBA6 for their supply of activated ubiquitin. In particular, it will be important to determine if the residual ubiquitylation detected in $U B E 1$ ts cell lines relies on UBA6 [34]. Moreover, mechanistic insight is needed to clarify how ubiquitin and FAT10 compete for activation by the same E1 and how these ULMs mediate E2 selectivity. The structural basis enabling discrimination between such loading events also needs to be determined. This work will require the identification of E2 and E3 enzymes specific for FAT10 conjugation, an endeavor that will be facilitated by the recent discovery of UBA6 as the cognate E1 for FAT10. Conditional knockout mice are required to determine the biological function of UBA6. Such mice should help to address whether, as indicated by expression analysis, UBA6 has a special role in the testis. The early embryonic lethality of $U B A 6$-deficient mice probably results from a defect in ubiquitin, rather than FAT10 activation, because FAT10-deficient mice are viable and healthy but are hypersensitive to lipopolysaccharide. The identification of further phenotypes of FAT10-deficient mice, in addition to the substrates of FAT10-conjugation, will enable new insights into the physiological functions of this fascinating member of the ULM family.

\section{Acknowledgements}

We thank Ingrid Kassner for critical reading of the manuscript, and Qiong Tong, Kay Diederichs and Stefanie Fischer for their help with figure preparation. Work in our laboratories was supported by the German Research Foundation (grant GR 1517/2-3) and the Fritz Thyssen Foundation (grant Az. 10.05.1.145).

\section{References}

1 Ciehanover, A. et al. (1978) A heat-stable polypeptide component of an ATP -dependent proteolytic system from reticulocytes. Biochem. Biophys. Res. Commun. 81, 1100-1105

2 Hershko, A. and Ciechanover, A. (1998) The ubiquitin system. Annu. Rev. Biochem. 67, 425-479

3 Hanna, J. and Finley, D. (2007) A proteasome for all occasions. FEBS Lett. 581, 2854-2861

4 Galan, J.M. and Haguenauer-Tsapis, R. (1997) Ubiquitin Lys63 is involved in ubiquitination of a yeast plasma membrane protein. EMBO J. 16, 5847-5854

5 Deng, L. et al. (2000) Activation of the IкB kinase complex by TRAF6 requires a dimeric ubiquitin-conjugating enzyme complex and a unique polyubiquitin chain. Cell 103, 351-361

6 Huang, D.T. et al. (2004) Ubiquitin-like protein activation. Oncogene 23, 1958-1971

7 Hicke, L. et al. (2005) Ubiquitin-binding domains. Nat. Rev. Mol. Cell Biol. 6, 610-621

8 Kerscher, O. et al. (2006) Modification of proteins by ubiquitin and ubiquitin-like proteins. Annu. Rev. Cell Dev. Biol. 22, 159-180

9 Hipp, M.S. et al. (2005) FAT10, a ubiquitin-independent signal for proteasomal degradation. Mol. Cell. Biol. 25, 3483-3491

10 Prudden, J. et al. (2007) SUMO-targeted ubiquitin ligases in genome stability. EMBO J. 26, 4089-4101

11 Uzunova, K. et al. (2007) Ubiquitin-dependent proteolytic control of SUMO conjugates. J. Biol. Chem. 282, 34167-34175

12 Xie, Y. et al. (2007) The yeast HEX3-SLX8 heterodimer is a ubiquitin ligase stimulated by substrate sumoylation. J. Biol. Chem. 282, 3417634184

13 Kamitani, T. et al. (2001) Targeting of NEDD8 and its conjugates for proteasomal degradation by NUB1. J. Biol. Chem. 276, 46655-46660

14 Suzuki, K. and Ohsumi, Y. (2007) Molecular machinery of autophagosome formation in yeast, Saccharomyces cerevisiae. FEBS Lett. 581, 2156-2161

15 Pelzer, C. et al. (2007) UBE1L2, a novel E1 enzyme specific for ubiquitin. J. Biol. Chem. 282, 23010-23014

16 Jin, J. et al. (2007) Dual E1 activation systems for ubiquitin differentially regulate E2 enzyme charging. Nature 447, 1135-1138

17 Chiu, Y.H. et al. (2007) E1-L2 activates both ubiquitin and FAT10. Mol. Cell 27, 1014-1023

18 Johnson, E.S. et al. (1997) The ubiquitin-like protein Smt3p is activated for conjugation to other proteins by an Aos $1 \mathrm{p} / \mathrm{Uba} 2 \mathrm{p}$ heterodimer. EMBO J. 16, 5509-5519

19 Liakopoulos, D. et al. (1998) A novel protein modification pathway related to the ubiquitin system. EMBO J. 17, 2208-2214

20 Haas, A.L. et al. (1982) Ubiquitin-activating enzyme. Mechanism and role in protein-ubiquitin conjugation. J. Biol. Chem. 257, 2543-2548 
21 Bohnsack, R.N. and Haas, A.L. (2003) Conservation in the mechanism of nedd8 activation by the human AppBp1- Uba3 heterodimer. J. Biol. Chem. 278, 26823-26830

22 Huang, D.T. et al. (2007) Basis for a ubiquitin-like protein thioester switch toggling E1-E2 affinity. Nature 445, 394-398

23 Lois, L.M. and Lima, C.D. (2005) Structures of the SUMO E1 provide mechanistic insights into SUMO activation and E2 recruitment to E1. EMBO J. 24, 439-451

24 Walden, H. et al. (2003) Insights into the ubiquitin transfer cascade from the structure of the activating enzyme for NEDD8. Nature 422, 330-334

25 Szczepanowski, R.H. et al. (2005) Crystal structure of a fragment of mouse ubiquitin-activating enzyme. J. Biol. Chem. 280, 22006-22011

26 Dye, B.T. and Schulman, B.A. (2007) Structural mechanisms underlying posttranslational modification by ubiquitin-like proteins. Annu. Rev. Biophys. Biomol. Struct. 36, 131-150

27 Huang, D.T. et al. (2005) Structural basis for recruitment of Ubc12 by an E2 binding domain in NEDD8's E1. Mol. Cell 17, 341-350

28 Ciechanover, A. et al. (1981) Activation of the heat-stable polypeptide of the ATP-dependent proteolytic system. Proc. Natl. Acad. Sci. U. S. A. $78,761-765$

29 Ciechanover, A. et al. (1982) Covalent affinity purification of ubiquitin activating enzyme. J. Biol. Chem. 257, 2537-2542

30 Hatfield, P.M. et al. (1990) Cloning of ubiquitin activating enzyme from wheat and expression of a functional protein in Escherichia coli. J. Biol. Chem. 265, 15813-15817

31 Zacksenhaus, E. and Sheinin, R. (1990) Molecular cloning, primary structure and expression of the human X linked A1S9 gene cDNA which complements the ts A1S9 mouse L cell defect in DNA replication. EMBO J. 9, 2923-2929

32 McGrath, J.P. et al. (1991) UBA1: an essential yeast gene encoding ubiquitin-activating enzyme. EMBO J. 10, 227-236

33 Handley, P.M. et al. (1991) Molecular cloning, sequence, and tissue distribution of the human ubiquitin-activating enzyme E1. Proc. Natl. Acad. Sci. U. S. A. 88, 258-262

34 Ciechanover, A. et al. (1984) Ubiquitin dependence of selective protein degradation demonstrated in the mammalian cell cycle mutant ts 85 . Cell 37, 57-66

35 Finley, D. et al. (1984) Thermolability of ubiquitin-activating enzyme from the mammalian cell cycle mutant ts85. Cell 37, 43-55

36 Kulka, R.G. et al. (1988) A Chinese hamster cell cycle mutant arrested at G2 phase has a temperature-sensitive ubiquitin-activating enzyme, E1. J. Biol. Chem. 263, 15726-15731

37 Handley-Gearhart, P.M. et al. (1994) Rescue of the complex temperature-sensitive phenotype of Chinese hamster ovary E36ts20 cells by expression of the human ubiquitin-activating enzyme cDNA Biochem. J. 304, 1015-1020

38 Kay, G.F. et al. (1991) A candidate spermatogenesis gene on the mouse $\mathrm{Y}$ chromosome is homologous to ubiquitin-activating enzyme E1. Nature 354, 486-489

39 Mitchell, M.J. et al. (1992) Marsupial Y chromosome encodes a homologue of the mouse Y-linked candidate spermatogenesis gene Ube1y. Nature 359, 528-531

40 Odorisio, T. et al. (1996) Transcriptional analysis of the candidate spermatogenesis gene UBE1y and of the closely related Ube1x shows that they are coexpressed in spermatogonia and spermatids but are repressed in pachytene spermatocytes. Dev. Biol. 180, 336-343

41 Hatfield, P.M. et al. (1997) The ubiquitin-activating enzyme (E1) gene family in Arabidopsis thaliana. Plant J. 11, 213-226

$42 \mathrm{Gu}$, X. et al. (2007) Cloning and characterization of a gene encoding the human putative ubiquitin conjugating enzyme E2Z (UBE2Z). Mol. Biol. Rep. 34, 183-188

43 Bedard, N. et al. (2005) Mice lacking the UBC4-testis gene have a delay in postnatal testis development but normal spermatogenesis and fertility. Mol. Cell. Biol. 25, 6346-6354

$44 \mathrm{Liu}, \mathrm{Z}$. et al. (2005) Characterization of E3Histone, a novel testis ubiquitin protein ligase which ubiquitinates histones. Mol. Cell. Biol. 25, 2819-2831

45 Lin, H. et al. (2000) Divergent $\mathrm{N}$-terminal sequences target an inducible testis deubiquitinating enzyme to distinct subcellular structures. Mol. Cell. Biol. 20, 6568-6578
46 Walden, H. et al. (2003) The structure of the APPBP1-UBA3-NEDD8ATP complex reveals the basis for selective ubiquitin-like protein activation by an E1. Mol. Cell 12, 1427-1437

47 Liu, Y.C. et al. (1999) A MHC-encoded ubiquitin-like protein (FAT10) binds noncovalently to the spindle assembly checkpoint protein MAD2. Proc. Natl. Acad. Sci. U. S. A. 96, 4313-4318

48 Lee, C.G. et al. (2003) Expression of the FAT10 gene is highly upregulated in hepatocellular carcinoma and other gastrointestina and gynecological cancers. Oncogene 22, 2592-2603

49 Raasi, S. et al. (1999) A ubiquitin-like protein which is synergistically inducible by interferon- $\gamma$ and tumor necrosis factor- $\alpha$. Eur. J. Immunol. 29, 4030-4036

50 Raasi, S. et al. (2001) The ubiquitin-like protein FAT10 forms covalent conjugates and induces apoptosis. J. Biol. Chem. 276, 35334-35343

51 Knipscheer, P. et al. (2007) Noncovalent interaction between Ubc9 and SUMO promotes SUMO chain formation. EMBO J. 26, 2797-2807

52 Rudolph, M.J. et al. (2001) Crystal structure of molybdopterin synthase and its evolutionary relationship to ubiquitin activation. Nat. Struct. Biol. 8, 42-46

53 Duda, D.M. et al. (2005) Structural analysis of Escherichia coli ThiF. J. Mol. Biol. 349, 774-786

54 Lehmann, C. et al. (2006) Structure of the Escherichia coli ThiS-ThiF complex, a key component of the sulfur transfer system in thiamin biosynthesis. Biochemistry 45, 11-19

55 Lake, M.W. et al. (2001) Mechanism of ubiquitin activation revealed by the structure of a bacterial MoeB-MoaD complex. Nature 414, 325-329

56 Wang, C. et al. (2001) Solution structure of ThiS and implications for the evolutionary roots of ubiquitin. Nat. Struct. Biol. 8, 47-51

57 Fan, W. et al. (1996) Identification of seven new human MHC class I region genes around the HLA-F locus. Immunogenetics 44, 97-103

58 Bates, E.E. et al. (1997) Identification and analysis of a novel member of the ubiquitin family expressed in dendritic cells and mature B cells. Eur. J. Immunol. 27, 2471-2477

59 Canaan, A. et al. (2006) FAT10/diubiquitin-like protein-deficient mice exhibit minimal phenotypic differences. Mol. Cell. Biol. 26, 5180-5189

60 Ross, M.J. et al. (2006) Role of ubiquitin-like protein FAT10 in epithelial apoptosis in renal disease. J. Am. Soc. Nephrol. 17, 996-1004

61 Ren, J. et al. (2006) FAT10 plays a role in the regulation of chromosomal stability. J. Biol. Chem. 281, 11413-11421

62 Zhang, D.W. et al. (2006) p53 negatively regulates the expression of FAT10, a gene upregulated in various cancers. Oncogene 25, 23182327

63 Hipp, M.S. et al. (2004) NEDD8 ultimate buster-1L interacts with the ubiquitin-like protein FAT10 and accelerates its degradation. J. Biol. Chem. 279, 16503-16510

64 Schmidtke, G. et al. (2006) The UBA domains of NUB1L are required for binding but not for accelerated degradation of the ubiquitin-like modifier FAT10. J. Biol. Chem. 281, 20045-20054

65 Vijay-Kumar, S. et al. (1987) Structure of ubiquitin refined at $1.8 \mathrm{~A}$ resolution. J. Mol. Biol. 194, 531-544

66 Narasimhan, J. et al. (2005) Crystal structure of the interferon-induced ubiquitin-like protein ISG15. J. Biol. Chem. 280, 27356-27365

67 Yuan, W. and Krug, R.M. (2001) Influenza B virus NS1 protein inhibits conjugation of the interferon (IFN)-induced ubiquitin-like ISG15 protein. EMBO J. 20, 362-371

68 Desterro, J.M.P. et al. (1999) Identification of the enzyme required for activation of the small ubiquitin-like protein SUMO-1. J. Biol. Chem. $274,10618-10624$

69 Gong, L. et al. (1999) Molecular cloning and characterization of human AOS1 and UBA2, components of the sentrin-activating enzyme complex. FEBS Lett. 448, 185-189

70 Gong, L. and Yeh, E.T. (1999) Identification of the activating and conjugating enzymes of the NEDD8 conjugation pathway. J. Biol. Chem. 274, 12036-12042

71 Furukawa, K. et al. (2000) A protein conjugation system in yeast with homology to biosynthetic enzyme reaction of prokaryotes. J. Biol. Chem. 275, 7462-7465

72 Komatsu, M. et al. (2004) A novel protein-conjugating system for Ufm1, a ubiquitin-fold modifier. EMBO J. 23, 1977-1986

73 Mizushima, N. et al. (1998) A protein conjugation system essential for autophagy. Nature 395, 395-398 